\title{
Complications and recurrence rates of patients with Ehlers-Danlos syndrome undergoing ventral hernioplasty: a case series
}

\author{
L. F. Kroese ${ }^{1} \cdot$ E. H. Mommers ${ }^{2} \cdot$ C. Robbens ${ }^{3} \cdot$ N. D. Bouvy ${ }^{2} \cdot$ J. F. Lange ${ }^{1,4} \cdot$ F. Berrevoet $^{3}$
}

Received: 12 June 2017 / Accepted: 19 January 2018 / Published online: 31 January 2018

(c) The Author(s) 2018. This article is an open access publication

\begin{abstract}
Purpose Ventral hernia repair is one of the most frequently performed surgical procedures, though recurrences are common. Recurrence can be caused by impaired collagen formation or maturation; hence, patients with Ehlers-Danlos syndrome (EDS) are potentially at increased risk for hernia recurrence. EDS causes altered collagen metabolism, though little is known about the influence of EDS on ventral hernioplasty outcomes. This study aims to analyze these patients to report complication rates, recurrence rates, and, if possible, to give recommendations for surgical intervention.

Methods A retrospective analysis between January 2000 and January 2017 was performed in a university hospital Belgium (UZ Ghent). Data on baseline characteristics, primary surgery, and hernias were extracted from patients' medical charts. Noted endpoints were postoperative complications and recurrences.

Results Fourteen patients (50\% males) were included. Ten (71\%) had an incisional hernia and four (29\%) had a primary ventral hernia. Median age was 45 years (IQR 37.75-52.75), median BMI was 24.82 (IQR 22.43-26.87). Four patients (29\%) smoked, one patient (7.1\%) had diabetes mellitus, and five patients (36\%) had an aneurysm of the abdominal aorta. All patients underwent elective open hernioplasty with mesh reinforcement. Three patients (21\%) had a postoperative complication (two infections, one seroma). Recurrence rate was $7.1 \%$ (one patient).

Conclusions This series describes 14 patients with a median follow-up of 50 months and a recurrence rate of $7.1 \%$. The low recurrence rate could be explained by the use of large meshes that reinforce the entire midline to compensate for the reduced collagen strength in EDS patients.
\end{abstract}

Keywords Ventral hernia $\cdot$ Ehlers-Danlos $\cdot$ Hernioplasty $\cdot$ Recurrence $\cdot$ Complications

L. F. Kroese, and E. H. Mommers contributed equally and should both be considered as first author.

$\triangle$ L. F. Kroese

1.kroese@erasmusmc.nl

1 Department of Surgery, Erasmus MC, University Medical Center, Room Ee-173, PO BOX 2040, 3000 CA Rotterdam, The Netherlands

2 Department of Surgery, Maastricht University Medical Center, Maastricht, The Netherlands

3 Department of General and HPB Surgery and Liver Transplantation, Ghent University Hospital, Ghent, Belgium

4 Department of Surgery, Havenziekenhuis Rotterdam, Rotterdam, The Netherlands

\section{Introduction}

Incisional hernia formation is one of the most frequent complications after abdominal surgery with midline laparotomy, occurring in 11-20\% of all laparotomies in the general population [1]. When patients have risk factors (obesity, smoking or abdominal aortic aneurysm), this rate can increase up to $35 \%$ [2].

It is hypothesized that a disturbed balance between mature and immature collagen can be part of the underlying mechanism leading to incisional hernia formation. Klinge et al. explain recurrent hernia formation as a combined problem of biology and technique [3]. The human extracellular matrix consists of twenty different types of collagen, of which $95 \%$ is type I and III collagen [4]. Patients with recurrent ventral hernias have a decreased collagen I/III ratio. Collagen type I is mature, mechanically stable collagen, whereas collagen type III is immature, mechanically instable collagen [3]. 
Alongside the previously mentioned collagens, it has been hypothesized by Schumpelick et al. that tenascin, a family of glycoproteins, could be linked to hernia formation [5]. Given this mechanism, patients with an underlying connective tissue disease, such as Ehlers-Danlos syndrome (EDS), can be at risk for a higher recurrence rate after both ventral and incisional hernia repair [5-7]. EDS was first described in 1901 and the syndrome characterizes itself by a triad of skin hyperextensibility, joint hypermobility, and tissue fragility. Originally, EDS was divided into numbered subtypes. In 1998, the Villefranche classification scheme divided EDS into six subtypes, based on clinical features, biochemical and genetic findings, and mechanism of inherence: classic (type I and II), hypermobility (type III), vascular (type IV), kyphoscoliosis (type VI), arthrochalasia (type VIIA and VIIB), and dermatosparaxis (type VIIC) [8, 9]. Because of overlapping symptoms in these different subtypes, categorizing EDS is no easy task. Including all subtypes of EDS, the incidence is approximately one in 5000 people, of which the hypermobility subtype is most common [8].

The recently published international classification of the Ehlers-Danlos Syndrome describes the genetic basis for each type of EDS. The classical, vascular, and arthrochalasia types have been linked to either type I or type III collagen disorders $[4,10]$. The hypermobility type is linked to tenascin $\mathrm{X}$ alterations. Although not all types of EDS have been linked to a specific protein disorder, many surgeons fear a high recurrence rate following hernia repair in EDS patients because of similar collagen disorders associated with both EDS and hernia recurrence [5-7].

EDS can potentially influence every part of the body where connective tissue is present. The literature on the relationship between EDS and hernia development is scarce and only includes a few case reports. Despite the lack of evidence, many surgeons believe that EDS may have a negative effect on the clinical outcome of ventral hernioplasty in terms of both higher postoperative complication and recurrence rates. The aim of this retrospective case series is to evaluate outcomes of ventral hernioplasty in patients with Ehlers-Danlos syndrome, the primary outcome is hernia recurrence and the secondary outcome is postoperative complications.

\section{Methods}

A retrospective analysis of hospital registries between January 2000 and January 2017 was performed in a large university hospital in Ghent, Belgium (Ghent University Hospital). Before commencement of the study, ethical approval and approval of the Institutional Review Boards was obtained. The hospital central registry was searched using either ICD-10/ICD-9-CM or Diagnosis Treatment Codes (DBC) for collagen disorder (Q79.6/756.83) and 'hernioplasty' (0303.123/0303.124). Any patient with a history of EDS (any type) and a ventral abdominal hernioplasty was eligible for inclusion in this study. Patients who registered an objection for participation in scientific research in their medical chart were excluded. Follow-up was obtained from patients' medical charts. All EDS patients were seen 3 weeks postoperatively, as well as every 6 months hereafter.

\section{Data collection}

The following data were extracted from patients medical charts: baseline characteristics (age, sex, body mass index (BMI), smoking, medical history and type of Ehlers-Danlos), information about the hernia (date of diagnosis, type of hernia, size, primary/recurrent hernia, complaints), details regarding the surgical procedure (date of operation, elective/emergency procedure, type of procedure, mesh type and size and drain placement), postoperative data (postoperative complications (seroma, hematoma, surgical site infection, other infection, mesh explantation, and recurrence)), and follow-up (duration, number of outpatient clinic visits, readmissions, reoperations, and complaints). Hernia characteristics were reported using the European Hernia Classification of the European Hernia Society (EHS) [11]. All data were stored and analyzed in SPSS ${ }^{\circledR}$ for windows version 24, IBM corp. Armonk, NY, released 2013.

\section{Results}

A total of 14 patients (seven males, seven females), with a median age of 45 years (range 24-60 years) were included with diagnosis dates between June 2009 and July 2016. Median BMI was 24.82 (IQR 22.43-26.87). Four patients smoked (29\%), one patient $(7.1 \%)$ had diabetes mellitus, and five patients (36\%) had an aneurysm of the abdominal aorta. Ten patients (71\%) were ASA Class II and three patients (21\%) were ASA Class III.

Two patients (7.1\%) had the classic type Ehlers-Danlos, six patients (43\%) had the hypermobility type, and four patients (29\%) had the vascular type. Patient baseline characteristics are shown in Table 1.

\section{Hernia characteristics}

Ten patients (71\%) had an incisional hernia and four patients (29\%) had a primary hernia (see Fig. 1 for hernia characteristics). One of the incisional hernias was a recurrent hernia, previously treated with a Marlex ${ }^{\circledR}$ mesh. The median hernia length of the primary hernias was $2.5 \mathrm{~cm}$ (range $2.0-5.0 \mathrm{~cm}$ ), and the median width was $2.0 \mathrm{~cm}$ (range $1.5-3.0 \mathrm{~cm}$ ). The median hernia length of the incisional hernias was $5.0 \mathrm{~cm}$ 
Table 1 Patient characteristics

\begin{tabular}{ll}
\hline Patient characteristics & $N=14$ \\
\hline Age at operation, years (IQR) & $45(38-53)$ \\
Male (\%) & $7(50)$ \\
BMI, kg/m² (IQR) & $24.82(22.43-26.87)$ \\
Smoking (\%) & $4(29)$ \\
Diabetes mellitus (\%) & $1(7.1)$ \\
Abdominal aortic aneurysm (\%) & $5(36)$ \\
ASA Class (\%) & \\
I & $0(0)$ \\
II & $10(71)$ \\
III & $3(21)$ \\
IV & $0(0)$ \\
Unknown (\%) & $1(7.1)$ \\
Ehlers-Danlos type (\%) & \\
Classic (type I and II) & $2(7.1)$ \\
Hypermobility (type III) & $6(43)$ \\
Vascular (type IV) & $4(29)$ \\
Kyphoscoliosis (type VI) & $0(0)$ \\
Arthrochalasia (type VIIA and B) & $0(0)$ \\
Dermatosparaxis (type VIIC) & $0(0)$ \\
Unknown (\%) & $2(14)$ \\
Type of primary surgery (in case of incisional hernia, $n=10)$ \\
Gynecologic & 2 \\
Vascular & 4 \\
Gastric & 3 \\
Colorectal & 1 \\
\hline & \\
\hline &
\end{tabular}

$I Q R$ interquartile range, $B M I$ body mass index, ASA American Society of Anesthesiologists (range 0.6-25 cm), and the median width was $3.5 \mathrm{~cm}$ (range $0.8-15 \mathrm{~cm}$ ). None of the patients underwent any concomitant procedures, nor had they concomitant hernias in other locations.

\section{Surgical characteristics}

All hernia repair procedures were elective (see Table 2 for operation details). In all patients, open surgery was performed, one procedure (a recurrent hernia repair) was planned as a laparoscopic procedure, but converted into an open procedure because of severe adhesions in the abdominal cavity. All patients received mesh reinforcement in either onlay $(n=1,7 \%)$, sublay $(n=9,64 \%)$, preperitoneal ( $n=3,21 \%)$, or intraperitoneal $(n=1,7 \%)$ position. The onlay procedure was performed using an Adhesix ${ }^{\circledR}$ mesh, sublay repairs were performed with UltraPro ${ }^{\circledR}(n=7)$, Adhesix $^{\circledR}(n=1)$, or Rebound ${ }^{\circledR}(n=1)$ mesh. Preperitoneal repairs were performed using the Ultrapro ${ }^{\circledR}(n=1,33 \%)$, or Rebound $^{\circledR}(n=2,67 \%)$ mesh, and the intraperitoneal procedure was performed using a Dualmesh ${ }^{\circledR}$. Average mesh size was $399 \mathrm{~cm}^{2}$ (range $63-900 \mathrm{~cm}^{2}$ ), with an average length of $23 \mathrm{~cm}$ (range 7.0-35 cm), and width of $16 \mathrm{~cm}$ (range $8.0-30 \mathrm{~cm}$ ). All sublay repairs could be closed in the midline using the Rives-Stoppa technique without the need for additional procedures (component separation or other). Eleven patients $(79 \%)$ received a drain at the end of the procedure.

\section{Perioperative outcomes}

No intra-operative complications were recorded. Postoperative complications occurred in three patients $(21 \%)$

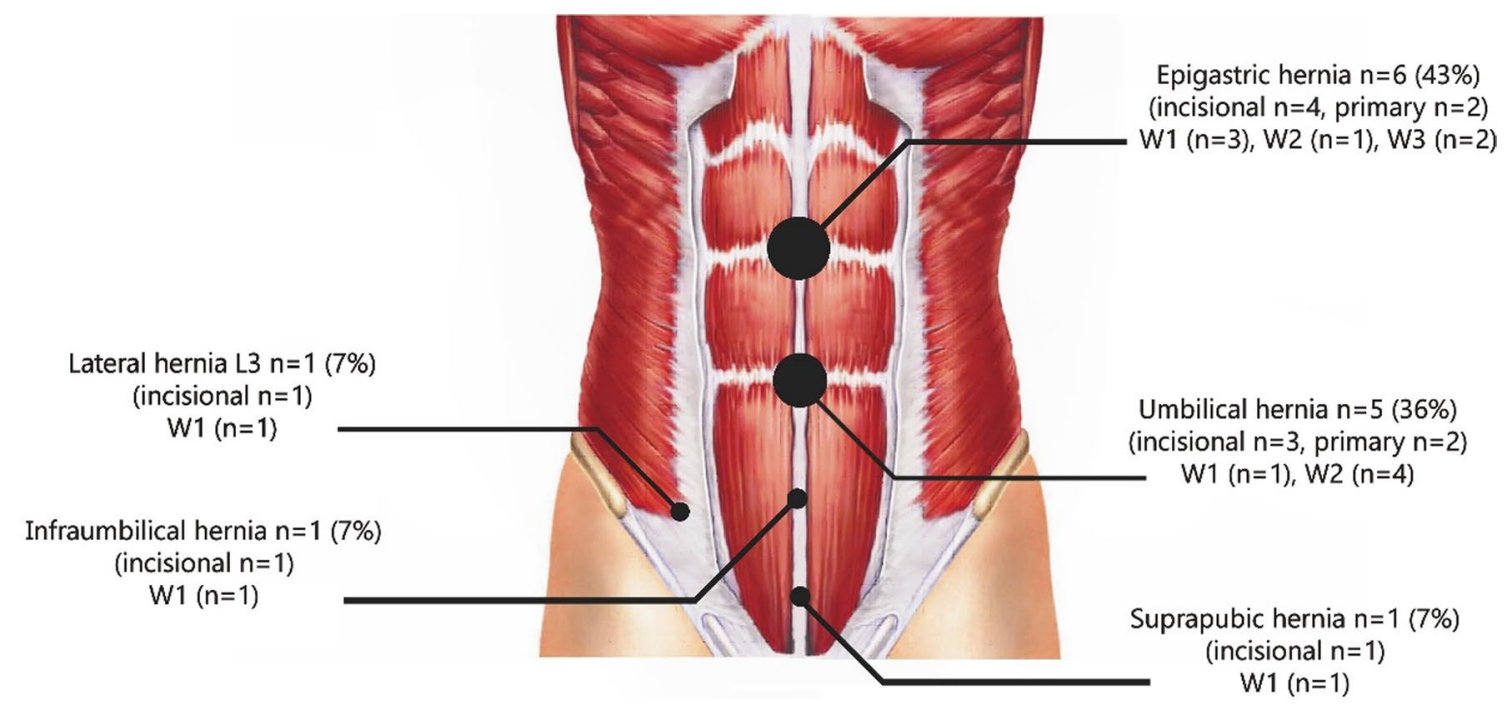

Fig. 1 Hernia characteristics. Hernia characteristics of all included patients. Characteristics are reported according to the European Hernia Society (EHS) guidelines for hernia classification. Width for inci- sional hernias $(W 1=<4 \mathrm{~cm} ; W 2 \geq 4-10 \mathrm{~cm} ; W 3 \geq 10 \mathrm{~cm}$ ), width for primary hernias $(W 1<2 \mathrm{~cm} ; W 2 \geq 2-4 \mathrm{~cm} ; W 3 \geq 4 \mathrm{~cm})$ 
Table 2 Surgical characteristics

\begin{tabular}{lc}
\hline Characteristic & $N=14$ \\
\hline Open procedure (\%) & $14^{\mathrm{a}}(100)$ \\
Emergency (\%) & $0(0)$ \\
Mesh location (\%) & \\
Onlay & $1(7.1)$ \\
Sublay/retromuscular & $9(64)$ \\
Preperitoneal & $3(21)$ \\
Intraperitoneal & $1(7.1)$ \\
Mesh type (\%) & \\
Ultrapro & $8(57)$ \\
Dualmesh & $1(7.1)$ \\
Adhesix & $2(14)$ \\
Rebound & $3(21)$ \\
Mesh size & \\
Length, cm (range) & $22.9(7.2-35)$ \\
Width, cm (range) & $15.8(8-30)$ \\
Surface (length $\times$ width), cm ${ }^{2}$ (range) & $399(63-900)$ \\
Drain placement $(\%)$ & $11(79)$ \\
Length of hospital stay, days (SD) & $3.38(1.04)$ \\
\hline
\end{tabular}

$S D$ standard deviation

${ }^{a}$ One procedure started as a laparoscopic procedure, but was converted into an open procedure

Table 3 Perioperative complications

\begin{tabular}{lc}
\hline Complication & $N=14$ \\
\hline No complications (\%) & $11(79)$ \\
Seroma (\%) & $1(7.1)$ \\
Hematoma (\%) & $0(0)$ \\
Surgical site infection (\%) & $2(14)$ \\
Other infection (\%) & $0(0)$ \\
Mesh explantation (\%) & $0(0)$ \\
Other (\%) & $0(0)$ \\
\hline
\end{tabular}

(Table 3). Mean hospital stay was 3.4 days ( \pm 1.04 days). One patient $(7 \%)$ had a seroma and two patients $(14 \%)$ had a surgical site infection. One of the patients with a surgical site infection had a BMI of $30.4 \mathrm{~kg} / \mathrm{m}^{2}$; the other patient had a relatively large hernia $(25 \times 15 \mathrm{~cm})$. One of the patients with a surgical site infection required antibiotic treatment and was, therefore, classified as a Clavien-Dindo Grade II complication [12]. The remaining two complications were Grade I. No other complications were recorded in the 30-day postoperative period.

\section{Long-term outcomes}

Median follow-up after surgery was 50 months (IQR 18-82, range 6-152 months). Patients visited the outpatient clinic a mean of three times (range 1-4 times). During this follow-up period, recurrences were assessed using clinical examination ( $n=3,29 \%)$, or clinical examination combined with ultrasonography $(n=10,71 \%)$. One patient $(7 \%)$ developed a hernia recurrence diagnosed by clinical examination. This patient was a 37-year-old female with EDS type III without relevant comorbidity or medical history (no diabetes, no aortic aneurysm, no smoking). The patient's BMI was 25.7. She presented with a primary umbilical hernia of $2 \times 2 \mathrm{~cm}$ (EHS Primary Abdominal wall Hernia Class: Midline Epigastric Medium hernia). She underwent an elective open repair with a preperitoneal mesh placement (Rebound ${ }^{\circledR} 9 \times 8 \mathrm{~cm}$ ) in August 2009. The procedure was without any complication. She developed a clinical recurrence after approximately 24-month follow-up, which resulted in esthetic complaints. The patient did not seek medical attention for her recurrence until 89-month follow-up, as she does not wish to undergo reoperation for the recurrence. No readmissions were performed. During follow-up, one patient died due to brain hemorrhage, unrelated to the hernioplasty procedure. For this patient, no long-term follow-up was available.

\section{Discussion}

This case series of 14 patients with Ehlers-Danlos syndrome (EDS) undergoing ventral hernia repair shows a $7.1 \%$ recurrence rate after a median follow-up period of 50 months. Current literature on ventral hernioplasty in EDS patients is scarce with only a hand full of case reports. Girotto et al. describes two patients with EDS and recurrent ventral abdominal wall hernias [13]. He treated these patients with a components separation technique and an onlay Marlex ${ }^{\circledR}$ mesh. Follow-up in this study is not described clearly. Fogel et al. describes a series of six ventral hernia repairs, of which two patients got a recurrent hernia, though important details regarding the surgical procedure and follow-up are not described as the focus of the article is on EDS and not on ventral hernia repair.

The current series is the first study that looks at patients with EDS as a specific risk group for developing hernia recurrence. Even though the detailed pathophysiology of incisional hernia formation is still illusive, many factors influence surgical wound healing and ultimately hernia formation [14]. One important factor is collagen synthesis and maturation. Given the well-established collagen impairment in EDS patients, a high recurrence rate in this population was anticipated. However, the $7 \%$ recurrence rate after open mesh repair in this study is lower than the $12 \%$ found in literature in the general patient population after elective open ventral hernioplasty with mesh reinforcement, after a median follow-up of 59 months [15]. The low recurrence rate can potentially be explained by the large mesh size. The average 
mesh size in this series was $16 \times 23 \mathrm{~cm}$, for an average hernia size of $3 \times 5 \mathrm{~cm}$. The 'oversized' mesh ensured a large surface for tissue ingrowth, which could compensate for the reduced collagen quality. Additionally, all patients were known to be diagnosed with EDS preoperatively. This might lead to a higher awareness of the surgeon. More conservative choices could have been made and more attention to the suturing technique could have been given. These factors, however, are hard to objectify.

During the 17-year inclusion period of this consecutive case series, only 14 patients were identified. There are several explanations for the relatively low number of EDS patients with ventral hernias. The most obvious one would be the low prevalence of EDS (1:5000). Another, more troubling, explanation would be identification failure of EDS in the outpatient hernia clinic. Since this case series is one of the first articles to discuss the potential influence of EDS on ventral hernia surgery outcomes, the problem may be underestimated or overlooked. Since EDS may be 'diagnosed' by one of many physicians either inside, or outside the hospital, central hospital registries may not always be up-to-date concerning the patient history. Hence, the treating physician must actively acquire information regarding EDS symptoms to not overlook the disease in the outpatient hernia clinic.

\section{Limitations}

A retrospective case series is methodologically unsuitable to determine the recurrence rate of ventral hernias in EDS patients accurately; hence, the percentages reported in this series must be interpreted with caution. Furthermore, because of the small sample size, detailed analysis of the relations between different types of EDS and clinical outcomes could not be made. Additionally, only two of the fourteen patients (14\%) had the classic EDS type. Although no in-depth analyses exist on the different subtypes of EDS and hernia recurrence, it could be hypothesized that the classic type would be more prone to recurrence than other types. This could partially explain the relatively low recurrence rate found in this study.

Finally, some patients had a relatively short follow-up period. This could potentially lead to an underreported recurrence rate.

Despite the abovementioned limitation, it is the authors' opinion that the following conclusions can be made based on the results of this article.

\section{Recommendations for hernioplasty in EDS patients}

- Establishing the diagnosis Ehlers-Danlos syndrome is the first step in providing tailored care for this complex patient population. If the family history or physical examination suggests Ehlers-Danlos syndrome, further examination is advised before attempting ventral abdominal wall hernioplasty.

- Treat 'small' ventral abdominal wall hernias as if they were bigger. The patients described in this series presented with relatively 'small' ventral hernias, though they were treated with a large (oversized) mesh and an extensive repair (most often Rives-Stoppa) with reinforcement along the entire midline or previous incision. Using large meshes provides a large surface for tissue ingrowth, which could compensate for the collagen impairment in EDS patients.

\section{Conclusion}

Patients with EDS are prevalent in the ventral abdominal wall hernia population. Identifying these patients is the first step towards tailored care. This series describes 14 patients with a median follow-up of 50 months and a recurrence rate of $7.1 \%$ (one patient). The low recurrence rate observed in this series might be explained by the use of a large mesh and reinforcement of the entire midline to compensate for the reduced collagen strength in EDS.

Acknowledgements No funding was received for this study.

Author contributions LK designed the study, analyzed and interpreted data and wrote the report. EM designed the study, analyzed and interpreted data and wrote the report. CR collected and interpreted data and revised the report. NB designed the study, interpreted data and wrote the report. JL designed the study, interpreted data and wrote the report. FB designed the study, interpreted data and wrote the report.

\section{Compliance with ethical standards}

Conflict of interest LK, EM, CR, NB, JL, and FB declare that they have no conflict of interest.

Ethical approval This study was approved by the local ethical committee.

Human and animal rights This article does not contain any studies with animals performed by any of the authors.

Informed consent For this type of article informed consent is not required.

Open Access This article is distributed under the terms of the Creative Commons Attribution-NonCommercial 4.0 International License (http://creativecommons.org/licenses/by-nc/4.0/), which permits any noncommercial use, distribution, and reproduction in any medium, provided you give appropriate credit to the original author(s) and the source, provide a link to the Creative Commons license, and indicate if changes were made. 


\section{References}

1. Deerenberg EB, Harlaar JJ, Steyerberg EW, Lont HE, van Doorn HC, Heisterkamp J, Wijnhoven BP, Schouten WR, Cense HA, Stockmann HB, Berends FJ, Dijkhuizen FP, Dwarkasing RS, Jairam AP, van Ramshorst GH, Kleinrensink GJ, Jeekel J, Lange JF (2015) Small bites versus large bites for closure of abdominal midline incisions (STITCH): a double-blind, multicentre, randomised controlled trial. Lancet 386(10000):1254-1260. https:// doi.org/10.1016/s0140-6736(15)60459-7

2. Muysoms FE, Detry O, Vierendeels T, Huyghe M, Miserez M, Ruppert M, Tollens T, Defraigne JO, Berrevoet F (2016) Prevention of incisional hernias by prophylactic mesh-augmented reinforcement of midline laparotomies for abdominal aortic aneurysm treatment: a randomized controlled trial. Ann Surg 263(4):638 645. https://doi.org/10.1097/sla.0000000000001369

3. Klinge U, Binnebosel M, Rosch R, Mertens P (2006) Hernia recurrence as a problem of biology and collagen. J Minim Access Surg 2(3):151-154

4. Radu P, Bratucu M, Garofil D, Goleanu V, Popa F, Strambu V (2015) The role of collagen metabolism in the formation and relapse of incisional hernia. Chirurgia (Bucur) 110(3):224-230

5. Rosch R, Junge K, Knops M, Lynen P, Klinge U, Schumpelick V (2003) Analysis of collagen-interacting proteins in patients with incisional hernias. Langenbecks Arch Surg 387(11-12):427-432. https://doi.org/10.1007/s00423-002-0345-3

6. Henriksen NA (2016) Systemic and local collagen turnover in hernia patients. Dan Med J 63(7):7

7. Antoniou GA, Georgiadis GS, Antoniou SA, Granderath FA, Giannoukas AD, Lazarides MK (2011) Abdominal aortic aneurysm and abdominal wall hernia as manifestations of a connective tissue disorder. J Vasc Surg 54(4):1175-1181. https://doi. org/10.1016/j.jvs.2011.02.065

8. Beighton P, De Paepe A, Steinmann B, Tsipouras P, Wenstrup RJ (1998) Ehlers-Danlos syndromes: revised nosology, Villefranche, 1997. Ehlers-Danlos National Foundation (USA) and Ehlers-Danlos Support Group (UK). Am J Med Genet 77(1):31-37. https:// doi.org/10.1136/ard.29.3.332
9. Callewaert B, Malfait F, Loeys B, De Paepe A (2008) Ehlers-Danlos syndromes and Marfan syndrome. Best Pract Res Clin Rheumatol 22(1):165-189. https://doi.org/10.1016/j.berh.2007.12.005

10. Malfait F, Francomano C, Byers P, Belmont J, Berglund B, Black J, Bloom L, Bowen JM, Brady AF, Burrows NP, Castori M, Cohen H, Colombi M, Demirdas S, De Backer J, De Paepe A, FournelGigleux S, Frank M, Ghali N, Giunta C, Grahame R, Hakim A, Jeunemaitre X, Johnson D, Juul-Kristensen B, Kapferer-Seebacher I, Kazkaz H, Kosho T, Lavallee ME, Levy H, Mendoza-Londono R, Pepin M, Pope FM, Reinstein E, Robert L, Rohrbach M, Sanders L, Sobey GJ, Van Damme T, Vandersteen A, van Mourik C, Voermans N, Wheeldon N, Zschocke J, Tinkle B (2017) The 2017 international classification of the Ehlers-Danlos syndromes. Am J Med Genet C Semin Med Genet 175(1):8-26. https://doi. org/10.1002/ajmg.c.31552

11. Muysoms FE, Miserez M, Berrevoet F, Campanelli G, Champault GG, Chelala E, Dietz UA, Eker HH, El Nakadi I, Hauters P, Hidalgo Pascual M, Hoeferlin A, Klinge U, Montgomery A, Simmermacher RK, Simons MP, Smietanski M, Sommeling C, Tollens T, Vierendeels T, Kingsnorth A (2009) Classification of primary and incisional abdominal wall hernias. Hernia 13(4):407414. https://doi.org/10.1007/s10029-009-0518-x

12. Dindo D, Demartines N, Clavien PA (2004) Classification of surgical complications: a new proposal with evaluation in a cohort of 6336 patients and results of a survey. Ann Surg 240(2):205-213

13. Girotto JA, Malaisrie SC, Bulkely G, Manson PN (2000) Recurrent ventral herniation in Ehlers-Danlos syndrome. Plast Reconstr Surg 106(7):1520-1526

14. Franz MG (2008) The biology of Hernia formation. The Surgical clinics of North America 88(1):1-7. https://doi.org/10.1016/j. suc.2007.10.007

15. Kokotovic D, Bisgaard T, Helgstrand F (2016) Long-term recurrence and complications associated with elective incisional hernia repair. JAMA 316(15):1575-1582. https://doi.org/10.1001/ jama.2016.15217 